\title{
METHODS TO GENERATE AN EXPECTED TURNING TRAFFIC FLOWS MATRIX FOR ROAD JUNCTION ANALYSIS
}

\author{
A. PRATELLI, L. SORDI, A. FARINA \\ DICI - Department of Civil and Industrial Engineering, University of Pisa, Italy
}

\begin{abstract}
In dependence upon a given geometric configuration, an actual or forecasted number of vehicles arriving at an intersection can turn left or right, otherwise proceed straight through it. This article can be placed in between a research working paper, and a kind of informational brief report. Indeed, it deals with forecasting techniques for estimation of intersection turning movements. Such issue is crucial, both in network planning and in traffic engineering, while its applications span from traffic safety and environmental impacts, to signal timing, roundabout design and setting of traffic control strategies. The number of vehicles making each movement on an existing intersection can be manually collected, especially when operational analyses are undertaken. Nevertheless, when the intersection is at the planning or design stage, an estimation process is required. In its first part, this paper provides a brief literature review of some of theoretical and practical methods focused to forecast the intersection turning movements. Such a review is limited on methods used to distribute the incoming and exiting traffic volumes within the different intersection branches, then generating an estimation of the so-called intersection O/D matrix of turning flows. The second part of the paper is experimental. Two different, but similar, heuristic procedures have described. Then, they have successively applied to some selected intersection real traffic data sets, and the respective computational performances were compared. Namely, the first one is known as proportion methods, while the second one is called as the difference, or deviation, method. Each method of these two starts from an initial matrix, and through iterative steps it reaches the best estimate of the matrix of turning flows, with respect to a given distribution model. Test intersections with their related set of real traffic data have been used as input, and each of the two procedures, as described in advance, was applied to the same numeric instances. The obtained values were compared in respect to few selected performance indicators. Finally, the computational results were displayed and discussed. On this basis, some insights are drawn and useful remarks for application and future research have been addressed.
\end{abstract}

Keywords: path traffic flows o/d matrix; road junction mathematical model; turning movement estimation.

\section{INTRODUCTION}

For over 50 years, many researchers and scholars have dealt with the problem of estimating the intersection turning movements. This can be achieved by generating the expected origin/ destination, or $\mathrm{O} / \mathrm{D}$, matrix of vehicles arriving at a given intersection and turn left or right, otherwise proceed straight through. Such estimation has built by starting with a range of traffic input data, including:

(a) Forecasts on turnover volumes in future years.

(b) Directional and non-directional forecasts of traffic volumes. The former are distinguished from the latter as they involve turning manoeuvres in every single direction, unlike the latter which do not concern the direction of travel.

(c) Data on current turning manoeuvres.

(d) Assignments regarding current turning manoeuvres.

(e) Observation time period.

(f) Number of branches at the intersection. 
The need to create a cheaper and faster O/D matrix was welcomed in the 1970s, when theoretical approaches fell apart and are replaced by methods based on simple information regarding the volumes of traffic arriving at intersections. In most urban areas, the data necessary for estimating the O/D matrix is found quite cheaply through the use of detectors.

Basically, there are the following two approaches that allow matrix estimation.

1. Parameter calibration techniques.

2. Methods of estimating the origin-destination matrix.

The first method uses linear or non-linear regression to build demand models by assuming a gravity flow model to estimate income in the matrix. This technique requires regional data, which are more difficult to find and vary over time, thus making it of limited practical use.

The second method is based only on traffic data at the intersection and on previous information entered in a matrix, this data is easily found and implemented without major obstacles. This technique can be implemented through two approaches: the first is based on statistical estimation procedures, the second instead concerns mathematical programming methods, based on the theory of maximum and minimum entropy and on equilibrium techniques in the transport network. Statistical estimation methods, on the other hand, use Bayes' theorem, which allows to produce a future evaluation based on a priori information, which could be linked to the searched data.

\section{PREVIOUS WORKS}

\subsection{Theoretical approaches}

In 1978 Willumsen [1] presents the maximum entropy approach following Wilson [2]. In the same year, Van Zuylen [3] discusses the principles of information minimization suggesting a method based on adding as little information as possible to the matrix of the initials of the points of turning point. Models that follow both philosophies in a combined way are developed and analysed in the following years, in fact this happens in 1980, thanks to the joint work of Van Zuylen and Willumsen [4]. Such model was based on the hypothesis of proportional assignment, in which some coefficients, determined exogenously, establish a proportion between each source/destination pair that uses a specific bond. This happens for every connection in the network. Many researchers have subsequently conducted tests and proposed improvements for this type of model, even Fisk has shown how to combine the theory of maximum entropy with the theory of the 'optimality' [5].

Figure 1 represents a 4-arm intersection and can be interpreted with the following notation:

- $O i=$ volume of traffic entering the intersection through branch $i$;

- $D j=$ volume of traffic exiting the intersection via branch $j$;

- $F i j=$ flow from branch $i$ to branch $j$;

- $P i j=$ a-priori probability that a vehicle at an intersection goes from $i$ to $j$.

The proposed problem can be solved by finding such set of Fij flows that satisfy the following constraints:

$$
\sum_{i} F_{i j}=D_{j}
$$




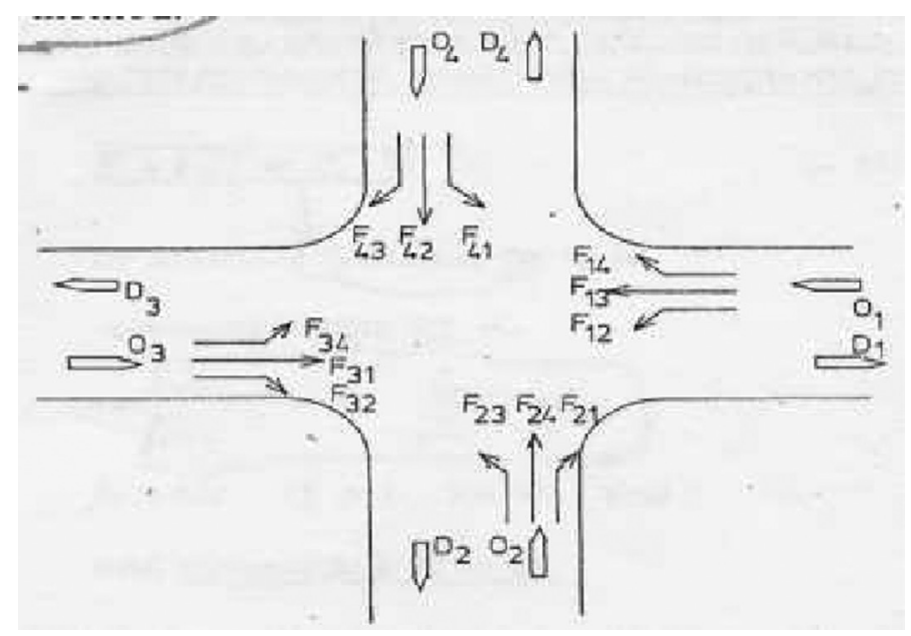

Figure 1: Typical 4-arm intersection turning diagram.

$$
\sum_{j} F_{i j}=O_{i}
$$

Equations (1) and (2) represent a system of 8 linear equations in 12 unknowns, assuming that U-turns are not allowed. The little information available apparently makes it impossible to determine a one-size-fits-all solution to the problem, yet traffic engineers often have more information than is contained in eqns (1) and (2). They know, for example, that turning streams at an intersection are less than streams that go straight on. Such empirical knowledge can be formulated as a priori probability of an event $(P i j)$, where $P i j$ represents the probability that a vehicle at an intersection will move from branch $i$ to $j$.

This way, Currin presented thoroughly the process of manual turning movement count at road intersections [6]. The method was suited to a road-crossing with twelve possible turning movements (no U-turns).

Over the last decades, many other researchers attempted to estimate the traffic flows matrix at road junctions and a number of both theoretical and heuristic methods were developed using traffic flows entering and leaving an intersection. Annotated references are the papers of Jeffreys and Norman [7], Razouki and Jadaan [8] and Razouki [9]. While the procedure of Eisenman and List [10] considers a roundabout and makes an estimation of turning movements using spot information on single vehicle's crossing trajectories. Jadaan [11] examined the accuracy of a road junction O/D matrix determined on traffic counts, and accepted a relative difference between actual and estimated flow of $13.1 \%$ for traffic flows of about $170 \mathrm{pcph}$.

A well-known theoretical method is the one proposed by Yang et al. [12]. They considered the problem of estimating the matrix of turning movements in the case of congested traffic, having available partial data regarding the connection flows, using an approach based on two levels. The upper level looks for a non-negative turn matrix that minimizes a weighted sum of the squares of the standard deviations from an initial O/D matrix, plus a deviation term that respects the observed traffic flows, which are determined in the second level, in accordance with the Wardrop's principles of network equilibrium [13].

There are also alternative methods that involve programming on two levels, one of these, developed by Fisk [14], involves using the upper level to construct an objective function, 
based on the principles of entropy, and the lower level containing equilibrium conditions, such as the variational inequality of McGuire and Winsten [15].

From a theoretical point of view, the problem of determining the O/D matrix at road intersections requires to establish the manoeuvres performed by the vehicles, having only the traffic volumes available and having to predict drivers behaviour. To do so, the estimation task can be formulated as a linear programming problem, even if it requires several data inputs but formally reaches to exact and accurate solutions. In this way, Sherali [16] proposed a linear programming based approach, which is characterized by a burdensome computational process that deeply limits its application to the design and planning field of road intersections.

\subsection{Heuristic methods}

Theoretical methods based on mathematical programming formulations require complicated algorithms to solve practical road junction instances.

Nevertheless, heuristic methods were designed according to simple algorithms, which lead to less accuracy but easier computational forecasts. Heuristic, or pure iterative, methods are also proposed for the estimation of the turning flow matrix, which have resulted to be quite effective in practical applications. Indeed, heuristics have been positively tested not only on road junctions, but also on larger transport networks.

Marshall [17] proposed a reducing criteria of the required number of turning flow counts. Only the one-way volumes into and out of the intersection are necessary. The method reduces the need for observers to count turning traffic, but the problem of forecasting the turning flows O/D matrix is not addressed.

In Report n.255 by NCHRP - National Cooperative Highway Research Program [18] there is a fairly good review of some iterative procedures for producing either directional or nondirectional turning flows through iterative steps. All these procedures need to start from an initial turning movements matrix, where the turning proportion, or probabilities, can be both a-priori assumed, and estimated analysing observed values or by considering traffic movements distribution at adjacent intersections.

For example, the initial matrix needs the so-called Directional Volume Method, founded on a basic iterative process developed by Furness [19] in his pioneering work, and adapted to intersection movements by Mekky [20]. The following iterative steps of the procedure try to balance the inflows (row totals) in respect to outflows (column totals) of a current turning flows matrix, until the differences of flows reach an acceptable approximation level. When there are still large differences from a step to the following, then several iteration might be necessary to reach the convergence of the inflow and outflow values.

\section{APPLIED FRAMEWORK}

Among the methods discussed in the previous section we can distinguish two categories: theoretical methods and practical methods. Within the first category, theoretical methods demand precise data and give solutions with a very good approximation. The limitation of these approaches concerns the variability of traffic data during the hours of a day and, also between the days in a week or in a month. This situation does not permit to have exact data that describe phenomena, the risk is to choose maximum or minimum values of flow rates that overestimate or underestimate the O/D matrix. The solution for this problem is to use the arithmetic mean between available traffic values observed in different hours of a day. This procedure appears onerous, in fact many measurements are necessary and then the collected data must be processed to obtain an average value. 
In computational experiences we are going to use practical methods. These approaches are different from the previous ones because they adapt themselves to the variability of traffic. Other differences involve mathematical laboriousness: the theoretical methods are based on maximum entropy and minimum information theory, practical ones use simple iterative processes.

Amongst the practical methods we are going to analyse the Ratio Method and the Delta method. Both methods are developed using the measured values of incoming and outgoing flow rates from the different branches of the roundabout. These values are crucial to generate for the first time the turning flow matrix by means of this relationship:

$$
x_{i j}=Q_{\mathrm{out} j} \frac{Q_{\mathrm{in} i}}{\sum_{j} Q_{\mathrm{in} i}} .
$$

This matrix has to be adjusted in order to satisfy the stopping rule. The adjustment concerns firstly row iterations and then column iterations. The iterative process repeats periodically until the matrix converges. In the Delta Method the adjustment takes place through two steps:

Step 1: Row iteration

$$
x_{i j}=x_{i j-1}+\left(\Delta_{e i} * \frac{X_{i j-1}}{Q_{\text {in calculated }}}\right), \Delta_{e i}=Q_{\text {in measured }}-Q_{\text {in calculated }}
$$

\section{Step 2: Column iteration}

$$
x_{i j}=x_{i j-1}+\left(\Delta_{u i} * \frac{x_{i j-1}}{Q_{\text {out calculated }}}\right), \Delta_{u i}=Q_{\text {out measured }}-Q_{\text {out calculated }}
$$

In the ratio method iterative steps are different and they are characterized by the following relationships:

Step 1: Row iteration

$$
x_{i j}=x_{i j-1} * \frac{Q_{e, \text { measured }}}{Q_{e, \text { calculated }}}
$$

Step 2: Column iteration

$$
x_{i j}=x_{i j-1} * \frac{Q_{u, \text { measured }}}{Q_{u, \text { calculated }}}
$$

Another difference relates to the stopping rule, in Delta method it is given by:

- For incoming flows: $\max \left(\frac{\Delta_{e i}}{Q_{e, \text { calculated }}}\right)<\varepsilon$

- For outgoing flows: $\max \left(\frac{\Delta_{u i}}{Q_{u, \text { calculated }}}\right)<\varepsilon$ 
In the ratio method the following applies:

$$
\text { Mean deviation }<\varepsilon
$$

Both methods present the threshold $\varepsilon$ that is determined in function of the desired accuracy.

\section{COMPUTATIONAL EXPERIENCES}

In this chapter some practical experiences using Delta Method and Ratio Method are analysed. In the first instance the O/D matrix in a roundabout with four branches is estimated; in this intersection the incoming and outgoing flow rates are known, these data are shown in Table 1 and Fig. 2.

Using these data and developing them with the previous eqns (4)-(5), it is possible to form the first $\mathrm{O} / \mathrm{D}$ matrix (Table 2), which is assembled in this way for the first row

$$
\begin{gathered}
x_{11}=0 \mathrm{vph} \\
x_{12}=596 * \frac{480}{480+1026+850}=121 \mathrm{vph} \\
x_{13}=1068 * \frac{480}{480+543+850}=274 \mathrm{vph} \\
x_{14}=750 * \frac{480}{480+543+1026}=176 \mathrm{vph}
\end{gathered}
$$

Following these equations for the other rows it is possible to obtain the initial matrix O/D (see Table 2).

Table 1: First tested intersection incoming and outgoing flow rates.

\begin{tabular}{lllll}
\hline$i / Q$ & $\mathbf{1}$ & $\mathbf{2}$ & $\mathbf{3}$ & $\mathbf{4}$ \\
\hline Qin & 480 & 543 & 1026 & 850 \\
Qout & 485 & 596 & 1068 & 750 \\
\hline
\end{tabular}

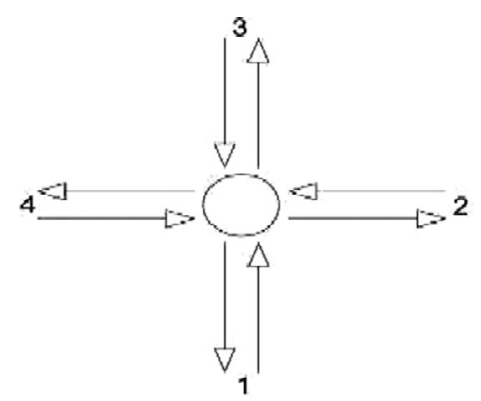

Figure 2: First tested intersection scheme. 
There are deviations on the rows, therefore it is necessary to distribute the errors by the first iterative step, as follows:

\section{Delta Method}

$$
\begin{array}{ll}
x_{11}=0 \mathrm{vph} & x_{11}=0 \mathrm{vph} \\
x_{12}=121+\left(-91 * \frac{121}{480}\right)=102 \mathrm{vph} & x_{12}=121 * 0.841=102 \mathrm{vph} \\
x_{13}=274+\left(-91 * \frac{274}{480}\right)=230 \mathrm{vph} & x_{13}=274 * 0.841=230 \mathrm{vph} \\
x_{14}=176+\left(-91 * \frac{176}{480}\right)=148 \mathrm{vph} & x_{14}=176 * 0.841=148 \mathrm{vph}
\end{array}
$$

Developing the calculations, we can note that the cells of the matrix contain the same values with Delta Method and Ratio Method, while the updated matrix has the form shown in Table 3.

It is now necessary to distribute the deviation on the columns through the second iteration step, following eqns (4) and (5) for the Delta Method, while (6) and (7) for the Ratio Method.

Table 2: First tested intersection initial O/D matrix.

\begin{tabular}{lllllll}
\hline & $\boldsymbol{1}$ & $\mathbf{2}$ & $\mathbf{3}$ & $\mathbf{4}$ & $Q_{\text {in.calculated }}$ & $Q_{\text {e.measured }}$ \\
\hline $\boldsymbol{1}$ & 0 & 121 & 274 & 176 & 571 & 480 \\
$\boldsymbol{2}$ & 109 & 0 & 310 & 199 & 617 & 543 \\
$\mathbf{3}$ & 206 & 260 & 0 & 376 & 841 & 1026 \\
$\mathbf{4}$ & 170 & 215 & 485 & 0 & 870 & 850 \\
$Q_{\text {out,calculated }}$ & 485 & 596 & 1068 & 750 & & \\
$Q_{\text {out,measured }}$ & 485 & 596 & 1068 & 750 & & \\
\hline
\end{tabular}

Table 3: First updated O/D matrix (at the end of the first iteration).

\begin{tabular}{lllllll}
\hline & $\mathbf{1}$ & $\mathbf{2}$ & $\mathbf{3}$ & $\mathbf{4}$ & $Q_{\text {u,calculated }}$ & $Q_{\text {in,measured }}$ \\
\hline $\boldsymbol{1}$ & 0 & 102 & 230 & 148 & 480 & 480 \\
$\mathbf{2}$ & 96 & 0 & 272 & 175 & 543 & 543 \\
$\mathbf{3}$ & 251 & 317 & 0 & 458 & 1026 & 1026 \\
$\mathbf{4}$ & 166 & 210 & 473 & 0 & 850 & 850 \\
$Q_{\text {out,calculated }}$ & 513 & 629 & 976 & 781 & & \\
$Q_{\text {in,measured }}$ & 485 & 596 & 1068 & 750 & & \\
\hline
\end{tabular}




\section{Delta Method}

$$
x_{11}=0 \mathrm{vph}
$$$$
x_{21}=96+\left(-28 * \frac{96}{513}\right)=90 \mathrm{vph}
$$$$
x_{31}=251+\left(-28 * \frac{251}{513}\right)=237 \mathrm{vph}
$$$$
x_{41}=166+\left(-28 * \frac{166}{513}\right)=157 \mathrm{vph}
$$

\section{Ratio Method}

$$
x_{11}=0 \mathrm{vph}
$$$$
x_{21}=96 * 0.945=90 \mathrm{vph}
$$$$
x_{31}=251 * 0.945=237 \mathrm{vph}
$$$$
x_{41}=166 * 0.945=157 \mathrm{vph}
$$

Applying such a formulation, we can complete the trip table and we obtain the values shown in Table 4.

We have to repeat these iterative steps until the matrix converges, it happens through four iterations with Ratio Method and through five iterations with Delta Method. Consequently, we can look at two different matrix solutions, and for the first Method the obtained matrix is displayed in Table 5.

This turning flow matrix converges because it respects the stopping rule, in fact:

$$
\text { Mean deviation }=\frac{0.0054+0.0064+0.0151+0.0107}{4}<\varepsilon
$$

where $\varepsilon$ is fixed this time in 0.01 .

In Delta Method the solution matrix is built in the way shown in Table 6.

This matrix converges because it respects eqns (8), (9) and (10). In fact, we can see from the Table 7 that errors are under the approximation threshold, fixed in 0.01 .

Table 4: First tested intersection O/D matrix after the second iteration.

\begin{tabular}{lllllll}
\hline & $\boldsymbol{1}$ & $\mathbf{2}$ & $\mathbf{3}$ & $\mathbf{4}$ & $Q_{\text {in,calculated }}$ & $Q_{\text {in,measured }}$ \\
\hline $\boldsymbol{1}$ & 0 & 97 & 252 & 142 & 491 & 480 \\
$\mathbf{2}$ & 90 & 0 & 298 & 168 & 556 & 543 \\
$\mathbf{3}$ & 237 & 300 & 0 & 440 & 978 & 1026 \\
$\mathbf{4}$ & 157 & 199 & 518 & 0 & 874 & 850 \\
$Q_{\text {out,calculated }}$ & 485 & 596 & 1068 & 750 & & \\
$Q_{\text {out,measured }}$ & 485 & 596 & 1068 & 750 & & \\
\hline
\end{tabular}

Table 5: First tested intersection O/D matrix obtained by the Ratio Method.

\begin{tabular}{lllll}
\hline & $\boldsymbol{1}$ & $\mathbf{2}$ & $\mathbf{3}$ & $\mathbf{4}$ \\
\hline $\boldsymbol{1}$ & 0 & 94 & 253 & 136 \\
$\boldsymbol{2}$ & 87 & 0 & 298 & 161 \\
$\mathbf{3}$ & 246 & 311 & 0 & 453 \\
$\mathbf{4}$ & 151 & 191 & 517 & 0 \\
\hline
\end{tabular}


Table 6: First tested intersection O/D matrix obtained by the Delta Method.

\begin{tabular}{lllll}
\hline & $\boldsymbol{1}$ & $\mathbf{2}$ & $\mathbf{3}$ & $\mathbf{4}$ \\
\hline $\boldsymbol{1}$ & 0 & 93 & 252 & 135 \\
$\boldsymbol{2}$ & 87 & 0 & 297 & 160 \\
$\mathbf{3}$ & 250 & 316 & 0 & 460 \\
$\mathbf{4}$ & 150 & 189 & 511 & 0 \\
\hline
\end{tabular}

Table 7: First tested intersection stopping rule for Delta Method.

\begin{tabular}{lllll}
\hline & $\mathbf{1}$ & $\mathbf{2}$ & $\mathbf{3}$ & $\mathbf{4}$ \\
\hline$Q_{\text {out,calculated }}$ & 487 & 598 & 1059 & 755 \\
$Q_{\text {out,measured }}$ & 485 & 596 & 1068 & 750 \\
$\Delta \mathrm{u}$ & 2 & -2 & 9 & -5 \\
Error & 0.003 & 0.004 & 0.008 & 0.007 \\
\hline
\end{tabular}

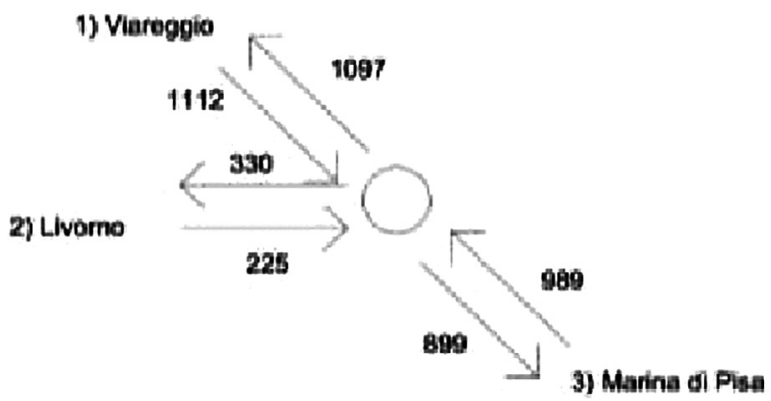

Figure 3: Scheme of the second tested intersection (3-branches roundabout).

Table 8: Design flow rates of the roundabout shown in Fig. 3.

\begin{tabular}{llll}
\hline $\mathrm{i} / \mathrm{Q}$ & $\mathbf{1}$ & $\mathbf{2}$ & $\mathbf{3}$ \\
\hline$Q_{\text {in }}$ & 1112 & 225 & 989 \\
$Q_{\text {out }}$ & 1097 & 330 & 899 \\
\hline
\end{tabular}

A different computational experience concerns a roundabout with three branches, which is located in the surroundings of Pisa (It). The design flow rates are at disposal again, and through these data it is possible to obtain turning flow matrix. The 3-branches roundabout scheme is shown in Fig. 3.

Developing calculations by eqns (4) and (5) the initial matrix is obtained; for the first row the values are the following:

$$
\begin{gathered}
x_{11}=0 \mathrm{vph} \\
x_{12}=330 * \frac{1112}{(1112+989)}=175 \mathrm{vph}
\end{gathered}
$$




$$
x_{13}=899 * \frac{1112}{(1112+225)}=748 \mathrm{veic} / \mathrm{h}
$$

Completing the correction of the other rows, the turning flow matrix is finally defined, as in Table 9.

The matrix obtained through the calculations needs to be corrected and the correction is possible by the steps previously introduced. We have to distribute deviations first on the rows and then on the columns, repeating this process with Ratio and Delta Method until the matrix approximation converges, we find the solution by means of 14 iterations with Ratio Method and 16 iterations through Delta Method. These approaches give the solution matrices shown in Tables 10 and 11.

The last experience analysed in this chapter is about a crossroads characterized by the scheme shown in Fig. 4.

This scheme contains incoming and outgoing flow rates. Then, it is possible to determine the initial matrix developing these last values, as follows:

$$
\begin{gathered}
x_{11}=0 \mathrm{vph} \\
x_{12}=500 * \frac{500}{(500+250+800)}=161 \mathrm{vph} \\
x_{13}=600 * \frac{500}{(500+450+800)}=171 \mathrm{vph} \\
x_{14}=600 * \frac{500}{(500+250+450)}=250 \mathrm{vph}
\end{gathered}
$$

Table 9: Second tested intersection turning flow matrix after the first iteration.

\begin{tabular}{llllll}
\hline & $\boldsymbol{1}$ & $\mathbf{2}$ & $\mathbf{3}$ & $Q_{\text {in,calculated }}$ & $Q_{\text {in,measured }}$ \\
\hline $\boldsymbol{1}$ & 0 & 175 & 748 & 922 & 1112 \\
$\mathbf{2}$ & 203 & 0 & 151 & 355 & 225 \\
$\mathbf{3}$ & 894 & 155 & 0 & 1049 & 989 \\
$Q_{\text {out,calculated }}$ & 1097 & 330 & 899 & & \\
$Q_{\text {out,measured }}$ & 1097 & 330 & 899 & & \\
\hline
\end{tabular}

Table 10: Second tested intersection Ratio Method solution matrix.

\begin{tabular}{llll}
\hline O/D 15 & $\boldsymbol{1}$ & $\mathbf{2}$ & $\mathbf{3}$ \\
\hline $\boldsymbol{1}$ & 0 & 243 & 843 \\
$\mathbf{2}$ & 172 & 0 & 56 \\
$\mathbf{3}$ & 925 & 87 & 0 \\
\hline
\end{tabular}


Table 11: Second tested intersection Delta Method solution matrix.

\begin{tabular}{llll}
\hline O/D 17 & $\boldsymbol{1}$ & $\mathbf{2}$ & $\mathbf{3}$ \\
\hline $\boldsymbol{1}$ & 0 & 246 & 845 \\
$\boldsymbol{2}$ & 174 & 0 & 54 \\
$\mathbf{3}$ & 923 & 84 & 0 \\
\hline
\end{tabular}

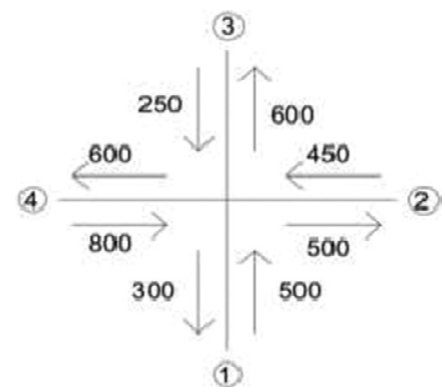

Figure 4: Scheme of the third tested intersection and its related traffic flow rates.

Table 12: Solution matrix for the third tested intersection.

\begin{tabular}{lllll}
\hline O/D 5 & $\boldsymbol{1}$ & $\mathbf{2}$ & $\mathbf{3}$ & $\mathbf{4}$ \\
\hline $\boldsymbol{1}$ & 0 & 130 & 140 & 233 \\
$\boldsymbol{2}$ & 78 & 0 & 141 & 234 \\
$\mathbf{3}$ & 44 & 74 & 0 & 133 \\
$\mathbf{4}$ & 177 & 296 & 319 & 0 \\
\hline
\end{tabular}

Following the iterative process, the matrix solution is obtained. This time the turning flow trip table is the same in Delta Method and in Ratio Method and it is defined by the values shown in Table 12.

\section{RESULTS AND DISCUSSION}

The computational experiences developed in the previous section can be analysed comparing the calculated values of flow rates to the observed ones. In order to do so, the following Figs. 5, 6 and 7 are set up.

The diagram of Fig. 5 shows the flow rate values in the first application roundabout: in the Delta Method the deviations are distributed on the rows and in the Ratio one on the columns. These results are respectively compared with the observed values and, in this specific case, we can note that the best matrix approximation is obtained by the Delta Method, even if the Ratio Method reaches the convergence first.

The graph of Fig. 6 shows that, in the second application roundabout, in both approaches the deviation is distributed on the rows and calculated values are close to the measured ones.

The graph of Fig. 7 is related to the third test intersection, i.e. the crossroads. In Fig. 7, it is shown that calculated values are the same, both with the Ratio and the Delta Method. 


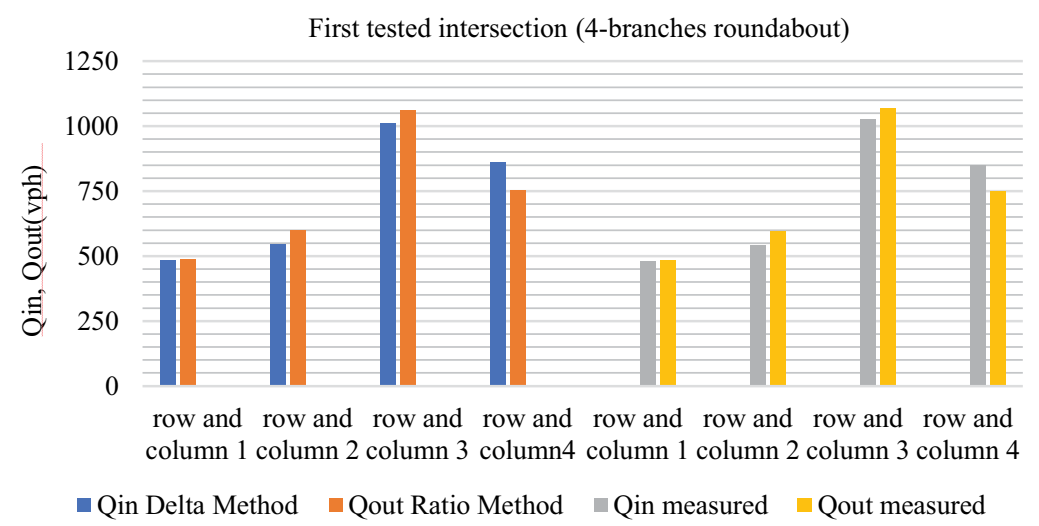

Figure 5: Results for the first tested intersection.

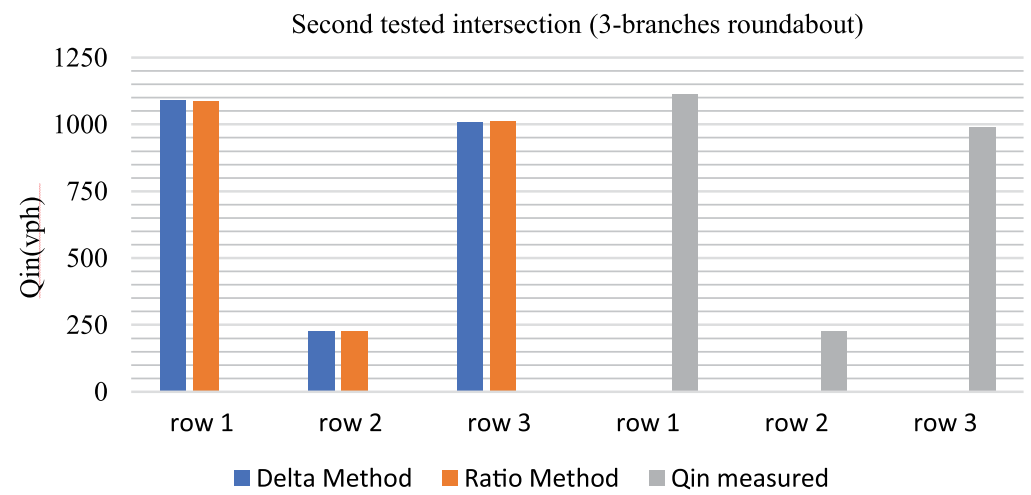

Figure 6: Results for the second tested intersection.

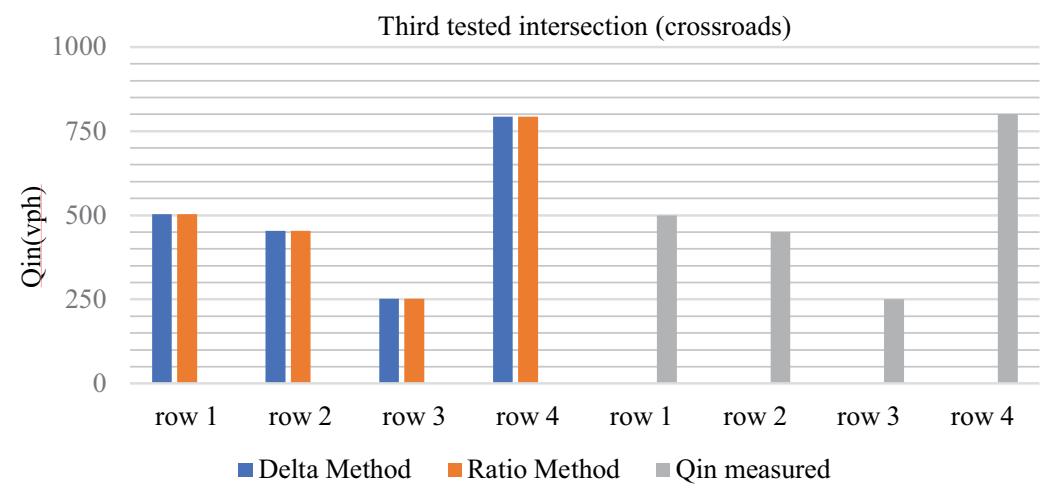

Figure 7: Results for the third tested intersection (crossroads). 


\section{SUMMARY AND CONCLUSIONS}

In this paper, some estimating methods have been analysed, we have divided them into two big categories: practical methods and theoretical ones. It was chosen to analyse Delta method and Ratio Method because they are quick, useful and easy to implement. Developing calculations in different types of intersection we have given some considerations. Both approaches, even if they use different iterative processes, give about the same turning flow matrix at the same iterative step, but the difference concerns the numbers of necessary iterations, useful to estimate O/D matrix. In our computational experiences Ratio Method needs fewer iterations than the other, but Delta one reaches a better approximation. These considerations are based on a limited number of cases and so they cannot be considered general. Finally, we have that both methods reach a satisfying accuracy but not excellent, achievable only through linear programming.

\section{REFERENCES}

[1] Willumsen, L.G., Estimation of $O-D$ matrix from traffic counts: a review, Working Paper 99, Institute for Transport Studies, University of Leeds, 1978.

[2] Wilson, A.G., Entropy in Urban and Regional Modelling, Routledge, London, 1970.

[3] Van Zuylen, H.J., A method to estimate a trip matrix from traffic volume counts. PTRC Summer Annual Meeting, University of Warwick, July 1978. PTRC Education \& Research Series Ltd, London, 1978.

[4] Van Zuylen, J.H. \& Willumsen, L.G., The most likely trip matrix estimated from traffic counts. Transportation Research Part B, 14, pp. 281-293, 1980.

[5] Fisk, C., On combining maximum entropy trip matrix estimation with user optimal assignment. Transportation Research Part B, 22(1), pp. 69-79, 1988.

[6] Currin, T.R., Introduction to traffic engineering, a manual for data collection and analysis, $2^{\text {nd }}$ Edition, Cengage Learning, UK, 2013.

[7] Jefferys, M. \& Norman, M., On finding realistic turning flows at road junctions. Traffic Engineering and Control, 18(1), pp.19-25, 1977.

[8] Razouki, S.S. \& Jadaan, K.S., Estimation of turning movements at three-arm rotaries. Dirasat Journal, Natural and Engineering Sciences, 24(2), pp. 305-312., 1997.

[9] Razouki, S.S., A mathematical model for determining the origin-destination matrix for five-arm rotaries. Journal of Engineering and Development, 4(3), pp. 86-99, 2000.

[10] Eisenman, S.M. \& List, G., A technique for data collection and estimation of turning movements at roundabouts, Proceedings of the 84th Annual Conference of the Transportation Research Board, Washington D.C., 2005.

[11] Jadaan, K., Accuracy of turning flow estimates at road junctions. ASCE Journal of Transportation Engineering, 115(4), pp. 438-449, 1989.

[12] Yang, H., Iida, Y \& Sasaki, T., Estimation of origin - destination matrices from traffic counts on congested networks. Transportation Research Part B, 26(6), pp. 417-434, 1992.

[13] Wardrop, J.G., Some theoretical aspects of road traffic research. Proceedings of the Institution of Civil Engineering, Part II, vol. 1, pp. 325-378, 1952.

[14] Fisk, C., Some developments in equilibrium traffic assignment. Transportation Research Part B, 14(3), pp. 243-255, 1980.

[15] Beckmann, M., McGuire, C.B., Winsten, C.B., Studies in the economics of transportation. Yale University Press, New Haven, Connecticut, U.S., 1956. 
[16] Sherali, H.D., Sivanandan, R., \& Hobeika, A.G., A linear programming approach for synthesizing origin - destination trip tables from link traffic volumes. Transportation Research Part B, 28(3), pp. 213-234, 1994.

[17] Marshall, M.L., Labour-saving Methods for Counting Traffic Movements at Three and Four-arm Junctions. Traffic Engineering and Control, 20(4), pp. 159-162, 1979.

[18] Pedersen, N.J., \& Samdahl, D.R., Highway traffic data for urbanized area project planning and design. 255th National Cooperative Research Program Report, Transportation Research Board, Washington D.C., U.S., 1982.

[19] Furness, K.P., Time Function Iteration, Traffic Engineering and Control, 7(7), pp. 458-460, 1965.

[20] Mekky, A. On Estimating Turning Flows at Road Junctions. Traffic Engineering and Control, 20(10), pp. 486-487, 1979. 